\title{
Symbolic Computation with Monotone Operators
}

\author{
Florian Lauster • D. Russell Luke . \\ Matthew K. Tam
}

\author{
July 19, 2018 \\ Dedicated to the memory of Jonathan Michael Borwein
}

\begin{abstract}
We consider a class of monotone operators which are appropriate for symbolic representation and manipulation within a computer algebra system. Various structural properties of the class (e.g., closure under taking inverses, resolvents) are investigated as well as the role played by maximal monotonicity within the class. In particular, we show that there is a natural correspondence between our class of monotone operators and the subdifferentials of convex functions belonging to a class of convex functions deemed suitable for symbolic computation of Fenchel conjugates which were previously studied by Bauschke \& von Mohrenschildt and by Borwein \& Hamilton. A number of illustrative examples utilizing the introduced class of operators are provided including computation of proximity operators, recovery of a convex penalty function associated with the hard thresholding operator, and computation of superexpectations, superdistributions and superquantiles with specialization to risk measures.
\end{abstract}

Keywords monotone operator, symbolic computation, experimental mathematics

Mathematics Subject Classification (2010) 47H05, 47N10, 68W30

\section{Introduction}

The Fenchel conjugate and the subdifferential of a function are two objects of fundamental importance in convex analysis. For this reason, software libraries or packages which have the ability to compute and manipulate such objects easily are a valuable edition to the convex analyst's toolkit. In the spirit of experimental

F. Lauster · D. R. Luke · M. K. Tam $(\varangle)$

Institut für Num. und Angew. Mathematik, Universität Göttingen, 37083 Göttingen, Germany. E-mail: m.tam@math.uni-goettingen.de

F. Lauster

E-mail: florian.lauster@stud.uni-goettingen.de

D.R. Luke

E-mail: r.luke@math.uni-goettingen.de 
mathematics $[5,2,3,4]$, such software also enables researchers to use the machinery of convex analysis to test ideas and look for patterns. There are also potential pedagogical uses if one believes, as we do, that nonsmooth analysis could and should be a part of the traditional "calculus" cannon taught to high school and beginning Bachelor's level students.

There are at least two possible paradigms for computation which can be followed for the development of such a software library, namely, computations can be done numerically or symbolically. Roughly speaking, the former involves numerical evaluation of the object under consideration on a grid of points in the ambient space whilst the latter involves the manipulation objects through symbolic expressions with a Computer Algebra System (CAS). We focus on the second approach, the "symbolic paradigm". For further details regarding numerical convex analysis, we refer the reader to $[15,16,1]$.

It is not too difficult to imagine that there are convex functions which, if not impossible, are too complex to represent and manipulate symbolically. Nevertheless, by restricting oneself to a suitable class of convex functions, a great deal can still be accomplished. Such a framework for symbolic convex analysis was proposed by Bauschke \& von Mohrenschildt $[7,8]$ for functions on the real line and an extension which could handle a many-dimensional setting was later proposed by Borwein \& Hamilton [14,10]. By "suitable class" we mean a class of functions which are representable by an appropriate data-structure, are closed under operations such as Fenchel conjugation and are sufficiently generic so as to capture many important examples.

As the subdifferential of a convex function is a monotone operator, it is natural to ask what class of monotone operators is suitable for symbolic computation as well as their relationship to the "suitable class" of convex functions studied in $[7,8,14,10]$. To the best of our knowledge, little has been done in this direction with the aforementioned works not focusing on the structure of the underlying monotone operators directly. In this work, we propose and study such a class of monotone operators which are suitable for implementation within a CAS. Among our main results, we prove that there is a natural correspondence between our class of monotone operators and the subdifferentials of the functions studied by Bauschke \& von Mohrenschildt (Theorem 3.1). We show that a consequence of this is that the class is closed under addition, scalar multiplication, taking inverses and taking resolvents (Proposition 3.3). We demonstrate the application of this class of operators on several illustrative examples in Section 4.

\section{Preliminaries}

Our notation and terminology are standard and can be found, for instance, in [11] and [22]. Since this work concerns computer implementations, we restrict our attention to $\mathbb{R}^{n}$ equipped with standard dot-product, denoted $\langle\cdot, \cdot\rangle$. For quick reference, we list some well-known facts from convex analysis that will be important later.

The (effective) domain of a function $f: \mathbb{R}^{n} \rightarrow[-\infty,+\infty]$ is the set $\operatorname{dom} f:=$ $\left\{x \in \mathbb{R}^{n}:|f(x)|<+\infty\right\}$. We will be interested in proper (not everywhere infinite and nowhere equal to $-\infty$ ), lower semi-continuous (lsc), convex functions. The subdifferential of a convex function is the set-valued mapping $\partial f: \mathbb{R}^{n} \rightrightarrows \mathbb{R}^{n}$ given 
by

$$
\partial f(\bar{x}):= \begin{cases}\left\{\phi \in \mathbb{R}^{n}:\langle\phi, x-\bar{x}\rangle \leq f(x)-f(\bar{x})\right\} & \bar{x} \in \operatorname{dom} f \\ \emptyset & \bar{x} \notin \operatorname{dom} f .\end{cases}
$$

The Fenchel conjugate of $f$ is the function $f^{*}: \mathbb{R}^{n} \rightarrow[-\infty,+\infty]$ defined by

$$
f^{*}(y):=\sup _{x \in \mathbb{R}^{n}}\{\langle y, x\rangle-f(x)\}
$$

The subdifferentials of a function and its Fenchel conjugate are inversely related.

Fact 2.1 ([11, Prop. 4.4.5]) Let $f: \mathbb{R}^{n} \rightarrow(-\infty,+\infty]$ be a function with $\bar{x} \in \operatorname{dom} f$. If $\bar{v} \in \partial f(\bar{x})$ then $\bar{x} \in \partial f^{*}(\bar{v})$. Conversely, if $f$ is a convex function which is lsc at $\bar{x}$ and $\bar{v} \in \partial f^{*}(\bar{x})$, then $\bar{v} \in \partial f(\bar{x})$.

Let $T: \mathbb{R}^{n} \rightrightarrows \mathbb{R}^{n}$ be a set-valued map. The domain of $T$ is the set $\operatorname{dom} T:=\{x \in$ $\left.\mathbb{R}^{n}: T x \neq \emptyset\right\}$, and the graph of $T$ is the set $\operatorname{gph} T:=\left\{(x, y) \in \mathbb{R}^{n} \times \mathbb{R}^{n}: y \in T x\right\}$. Recall that $T$ is monotone if

$$
\left.\begin{array}{l}
\left(x, x^{+}\right) \in \operatorname{gph} T \\
\left(y, y^{+}\right) \in \operatorname{gph} T
\end{array}\right\} \Longrightarrow\left\langle x-y, x^{+}-y^{+}\right\rangle \geq 0 .
$$

If the inequality in (1) is strict whenever $x \neq y$, then $T$ is said to be strictly monotone. If $T$ is monotone and there exists no monotone operator whose graph properly contains the graph of $T$, then $T$ is said to be maximal monotone.

Next we recall a notion stronger than monotonicity. An operator $T: \mathbb{R}^{n} \rightrightarrows \mathbb{R}^{n}$ is said to be cyclically monotone if, for every $n \geq 2$, it holds that

$$
\left.\begin{array}{c}
\left(x_{1}, x_{1}^{+}\right) \in \operatorname{gph} T \\
\vdots \\
\left(x_{n}, x_{n}^{+}\right) \in \operatorname{gph} T \\
x_{n+1}=x_{1}
\end{array}\right\} \Longrightarrow \sum_{i=1}^{n}\left\langle x_{i}-x_{i+1}, x_{i}^{+}\right\rangle \geq 0
$$

Analogously, if $T$ is cyclically monotone and there exists no cyclically monotone operator whose graph properly contains the graph of $T$, then $T$ is said to be maximal cyclically monotone.

The family of maximal cyclically monotone operators can be characterized as the subdifferentials of proper, lsc, convex functions.

Fact 2.2 (Rockafellar [21]) Let $T: \mathbb{R}^{n} \rightrightarrows \mathbb{R}^{n}$. Then $T$ is maximal cyclically monotone if and only if there exists a proper, lsc, convex function $f: \mathbb{R}^{n} \rightarrow(-\infty,+\infty]$ such that $T=\partial f$.

Regarding Fact 2.2, we remark that cyclic monotonicity is stronger than mere monotonicity. However, on the real-line (i.e., $\operatorname{dom} T=\mathbb{R}$ ), the two notions coincide [6, Th. 22.18].

In general, the sum of two maximal monotone operators need not be maximal unless an appropriate constraint qualification is satisfied. In the following Fact, we give one such example. 
Fact 2.3 (Maximal monotonicity of sums [6, Th. 24.3]) Let $T_{1}, T_{2}: \mathbb{R}^{n} \rightrightarrows \mathbb{R}^{n}$ be maximal monotone such that

$$
\text { cone }\left(\operatorname{dom} T_{1}-\operatorname{dom} T_{2}\right)=\operatorname{cl} \operatorname{span}\left(\operatorname{dom} T_{1}-\operatorname{dom} T_{2}\right) .
$$

Then $T_{1}+T_{2}$ is maximal monotone. In particular, (2) holds whenever $0 \in \operatorname{int}\left(\operatorname{dom} T_{1}-\right.$ $\left.\operatorname{dom} T_{2}\right)$.

Let $S$ be a subset of $\mathbb{R}^{n}$. Recall that $S$ is said to be an $m$-dimensional simplex if there exist a set of $m+1$ affinely independent points whose convex hull equals $S$. We say that $S$ is locally simplicial, in the sense of [20], if for each $x \in S$ there exists a finite collection of simplicies $S_{1}, \ldots, S_{m}$ contained in $S$ such that, for some neighborhood $U$ of $x$,

$$
U \cap\left(S_{1} \cup \cdots \cup S_{m}\right)=U \cap S .
$$

Examples of locally simplicial sets are line segments, polyhedral convex sets, and relatively open convex sets.

Fact 2.4 (Continuity on the effective domain [20, Th. 10.2]) Let $f$ be a convex function on $\mathbb{R}^{n}$, and let $S$ be any locally simplicial subset of $\operatorname{dom} f$. Then $f$ is upper semi-continuous relative to $S$. In particular, if $f$ is lsc, then $f$ is continuous relative to $S$.

As a consequence of Fact 2.4 and the fact that every convex subset of $\mathbb{R}$ is an interval (and hence simplicial), it follows that every convex lsc function on $\mathbb{R}$ is necessarily continuous on its domain.

\section{Operators in One Dimension}

Before turning our attention to monotone operators, we recall first the class of convex functions originally studied in $[7,8,14,10]$. The Maple package Symbolic Convex Analysis Toolbox (SCAT) [10] implements precisely this class of functions. Since the definition of the class is recursive in the dimension of the underlying domain, it is essential to understand the one-dimensional case first.

Recall that a function $f: \mathbb{R}^{n} \rightarrow[-\infty,+\infty]$ is strictly convex if

$$
f(\lambda x+(1-\lambda) y)<\lambda f(x)+(1-\lambda) f(y),
$$

for all $x, y \in \operatorname{dom} f$ with $x \neq y$ and for all $\lambda \in(0,1)$.

Definition 3.1 ( $\mathcal{F}$-functions $[\mathbf{7}, 8]$ ) For a set of finitely many points $A=\left\{a_{i}\right\}_{i=1}^{m}$ satisfying

$$
a_{0}=-\infty<a_{1}<\cdots<a_{m-1}<a_{m}=+\infty,
$$

we say a function $f: \mathbb{R} \rightarrow(-\infty,+\infty]$ belongs to $\mathcal{F}(A)$ if:

(a) $f$ is closed and convex;

(b) $f$ is continuous on its effective domain; and

(c) the restriction of $f$ to the interval $\left(a_{i}, a_{i+1}\right)$ is either (i) affine, (ii) strictly convex and differentiable, or (iii) identically equal to $+\infty$.

The class of functions $\mathcal{F}$ is the union of $\mathcal{F}(A)$ over all finite sets of points $A$ satisfying (3) 
Recalling that a function is closed (i.e., its epigraph is a closed set) if and only if it is lsc, we observe that Condition (a) is equivalent to requiring that functions in $\mathcal{F}$ be either proper, lsc and convex, or identically equal to $+\infty$. Moreover, as a convex function is continuous on the relative interior of its domain [20, Th. 10.1], the only place where Condition (b) can play a role is at boundary points of the effective domain.

Remark 3.1 The presentation of Definition 3.1 given here differs slightly from the version given in $[7,8]$ in that we introduce the set $\mathcal{F}$ through the union of the sets $\mathcal{F}(A)$ rather than directly.

One of the most important properties of $\mathcal{F}$-functions is that the class is closed under Fenchel conjugation. This is ensures that a data-structure designed to represent functions belonging to $\mathcal{F}$ is also able to represent their conjugates. This closure property was noted in [8] without proof. We shall return to this topic later where our soon to be introduced class of monotone operator to furnish a convenient proof. Another important property of the subdifferentials of $\mathcal{F}$-functions is that they may be expressed explicitly in terms of their gradient, when this exists.

Proposition 3.1 (Computing $\mathcal{F}$-subdifferentials $[10, \S 2.1 .2]$ ) Suppose $f \in \mathcal{F}(A)$ for $A=\left\{a_{i}\right\}_{i=1}^{m}$, and let $\left.f\right|_{i}$ (resp. $\left.\left.f^{\prime}\right|_{i}\right)$ denote the restriction of $f$ (resp. $\left.f^{\prime}\right)$ to the interval $\left(a_{i}, a_{i+1}\right)$. Then $\partial f$ can be piecewise defined according to the following three cases.

(a) If $x \notin \operatorname{dom} f$ then $\partial f\left(x_{0}\right)=\emptyset$;

(b) If $x \in \operatorname{int}(\operatorname{dom} f)$ then

$$
\partial f(x)=\left[\lim _{y \uparrow x} f^{\prime}(y), \lim _{y \downarrow x} f^{\prime}(y)\right] ;
$$

(c) If $x \in \operatorname{dom} f \backslash \operatorname{int}(\operatorname{dom} f)$ then $x=a_{i}$ for some $i \in\{1,2, \ldots, m-1\}$. In this case

$$
\partial f\left(a_{i}\right)= \begin{cases}(-\infty,+\infty) & \text { if }\left.f\right|_{i-1}=\infty=\left.f\right|_{i}, \\ \left(-\infty,\left.\lim _{y \downarrow a_{i}} f\right|_{i} ^{\prime}(y)\right] & \text { if }\left.f\right|_{i-1}=\infty \neq\left. f\right|_{i}, \\ {\left[\left.\lim _{y \uparrow a_{i}} f\right|_{i-1} ^{\prime}(y),+\infty\right)} & \text { if }\left.f\right|_{i-1} \neq \infty=\left.f\right|_{i} .\end{cases}
$$

As we have already seen, the subdifferential of proper, lsc, convex function is always a maximal (cyclically) monotone operator (Fact 2.2). Thus, in light of the above proposition, we collect some of the finer monotonicity properties of the subdifferentials of $\mathcal{F}$-functions. The following lemma will simplify the proof of Proposition 3.2.

Lemma 3.1 Let $f \in \mathcal{F}(A)$ be a proper function where $A=\left\{a_{i}\right\}_{i=0}^{m}$. Then the restriction of $\partial f$ to the interval $\left(a_{i}, a_{i+1}\right)$ is either (i) single-valued and constant, (ii) singlevalued, continuous and strictly monotone, or (iii) identically equal to the empty-set.

Proof Consider the restriction of $f$ to the open interval $\left(a_{i}, a_{i+1}\right)$. We distinguish three cases based on Definition 3.1(c). (i) If $f$ is affine on $\left(a_{i}, a_{i+1}\right)$ then $f^{\prime}$ is singlevalued and constant. (ii) If $f$ is differentiable on $\left(a_{i}, a_{i+1}\right)$ then $f^{\prime}$ is continuous on $\left(a_{i}, a_{i+1}\right)$ [20, Thm. 25.5.1], and if $f$ is strictly convex on $\left(a_{i}, a_{i+1}\right)$ then $f^{\prime}$ is strictly monotone on $\left(a_{i}, a_{i+1}\right)$, by [11, Exer. 2.1.14] and [22, Thm. 12.17]. (iii) Otherwise, by virtue belonging to $\mathcal{F}, f$ must be identically equal to $+\infty$ on $\left(a_{i}, a_{i+1}\right)$ and, by definition, its subdifferential is identically equal to the empty-set on $\left(a_{i}, a_{i+1}\right)$. 
Proposition 3.2 (Structure of $\mathcal{F}$-subdifferentials) Let $f \in \mathcal{F}(A)$ be a proper function where $A=\left\{a_{i}\right\}_{i=0}^{m}$. The following assertions hold.

(a) The restriction of $\partial f$ to the interval $\left(a_{i}, a_{i+1}\right)$ is either (i) single-valued and constant, (ii) single-valued, continuous and strictly monotone, or (iii) identically equal to the empty-set.

(b) For any $x \in \operatorname{dom} f \backslash \operatorname{int}(\operatorname{dom} f), x=a_{i}$ for some $i \in\{1, m-1\}$ and

$$
\left\{\begin{aligned}
\partial f\left(a_{i}\right) & =(-\infty,+\infty) & & \text { if }\left.f\right|_{i-1}=\infty=\left.f\right|_{i}, \\
\max \partial f\left(a_{i}\right) & =\left.\lim _{x \downarrow a_{i}} f\right|_{i} ^{\prime}(x) & & \text { if }\left.f\right|_{i-1}=\infty \neq\left. f\right|_{i}, \\
\min \partial f\left(a_{i}\right) & =\left.\lim _{x \uparrow a_{i}} f\right|_{i-1} ^{\prime}(x) & & \text { if }\left.f\right|_{i-1} \neq \infty=\left.f\right|_{i} ;
\end{aligned}\right.
$$

where, by convention, $\min \emptyset=+\infty$ and $\max \emptyset=-\infty$.

Proof (a): Follows by applying Lemma 3.1 to each interval $\left(a_{i}, a_{i+1}\right)$. (b): This is a direct consequence of Proposition 3.1(c).

Motivated by Propositions 3.1 and 3.2, we define the following class of monotone operators. We shall show that, in particular, it contains all subdifferentials of $\mathcal{F}$-functions.

Definition 3.2 ( $\mathcal{T}$-operators) For a set of finitely many points $B=\left\{b_{i}\right\}_{i=0}^{l}$ satisfying

$$
b_{0}=-\infty<b_{1}<\cdots<b_{l-1}<b_{l}=+\infty,
$$

we say a set-valued operator $T: \mathbb{R} \rightrightarrows \mathbb{R}$ belongs to $\mathcal{T}(B)$ if there exists a maximal monotone extension $\widetilde{T}$ of $T$ such that the restriction $\widetilde{T}$ to each interval $\left(b_{i}, b_{i+1}\right)$ is either

(i) single-valued and constant;

(ii) single-valued, continuous and strictly monotone; or

(iii) identically equal to the empty-set.

The class of operators $\mathcal{T}$ is the union of $\mathcal{T}(B)$ over all finite sets of points $B$ satisfying (4).

The proposition which soon follows establishes that the class of $\mathcal{T}$-operators is well-suited for symbolic manipulation. Moreover, as a monotone operator can have only countably many discontinuities in its domain, the restriction to monotone operators possessing at most finitely many discontinuities is still quite general.

Remark 3.2 ( $\mathcal{T}$-operators at points of discontinuity) Let $T \in \mathcal{T}$ with maximal monotone extension $\widetilde{T} \in \mathcal{T}(B)$ where $B=\left\{b_{i}\right\}_{i=1}^{l}$. From the definition of $\mathcal{T}$-operators, the only possible points of discontinuity of $T$ are the points in $B$. At a point $b_{i} \in \operatorname{int}(\operatorname{dom} \widetilde{T})$ for some $i \in\{1, \ldots, l-1\}$, the restriction of $\widetilde{T}$ to either of the open intervals $\left(b_{i-1}, b_{i}\right)$ and $\left(b_{i}, b_{i+1}\right)$ is continuous and hence, by monotonicity, both of the limits $\lim _{x \uparrow b_{i}} \widetilde{T}(x)$ and $\lim _{x \downarrow b_{i}} \widetilde{T}(x)$ are finite. We therefore have that

$$
T\left(b_{i}\right) \subseteq \widetilde{T}\left(b_{i}\right)=\left[\lim _{x \uparrow b_{i}} T(x), \lim _{x \downarrow b_{i}} T(x)\right],
$$

where the equality holds due to outer semi-continuity of $\widetilde{T}[12, \S 4.2]$. 
The following theorem shows that all of the most important closure properties hold for the class of $\mathcal{T}$-operators.

Proposition 3.3 (Properties of $\mathcal{T}$-operators) The following assertions hold.

(a) If $T \in \mathcal{T}$ and $\lambda \geq 0$ then $\lambda T \in \mathcal{T}$.

(b) If $T_{1}, T_{2} \in \mathcal{T}$ then $T_{1}+T_{2} \in \mathcal{T}$.

(c) If $T \in \mathcal{T}$ then $T^{-1} \in \mathcal{T}$.

(d) If $T \in \mathcal{T}$ and $\lambda>0$ then $(I+\lambda T)^{-1} \in \mathcal{T}$.

(e) If $T_{1}, T_{2} \in \mathcal{T}$ then $\left(T_{1}^{-1}+T_{2}^{-1}\right)^{-1} \in \mathcal{T}$.

Proof (a): Let $\widetilde{T} \in \mathcal{T}$ be a maximal monotone extension of $T$ and $\lambda \geq 0$. Then $\lambda \widetilde{T} \in \mathcal{T}$ and, moreover, $\lambda \widetilde{T}$ is a maximal monotone extension of $\lambda T$.

(b): Define $T:=T_{1}+T_{2}$ and let $\widetilde{T}_{1}$ and $\widetilde{T}_{2}$ denote maximal monotone extensions respectively of $T_{1}$ and $T_{2}$ contained in $\mathcal{T}$. Setting $\widetilde{T}:=\widetilde{T}_{1}+\widetilde{T}_{2}$, we therefore have that

$$
\operatorname{dom} \widetilde{T}=\operatorname{dom} \widetilde{T}_{1} \cap \operatorname{dom} \widetilde{T}_{2} \supseteq \operatorname{dom} T_{1} \cap \operatorname{dom} T_{2}=\operatorname{dom} T .
$$

We now distinguish three cases based on $\operatorname{dom} \widetilde{T}$.

(i) Suppose $\operatorname{dom} \widetilde{T}=\emptyset$. Then, using (5), it follows that $\operatorname{dom} T=\emptyset$. As the empty relation is trivially contained in $\mathcal{T}$, we have that $T \in \mathcal{T}$.

(ii) Suppose $\operatorname{dom} \widetilde{T} \neq \emptyset$ but $\operatorname{int}(\operatorname{dom} \widetilde{T})=\emptyset$. Since $\operatorname{dom} \widetilde{T}$ is the intersection of two convex sets, $\operatorname{dom} \widetilde{T}_{1}$ and $\operatorname{dom} \widetilde{T}_{2}$, it follows that $\operatorname{dom} \widetilde{T}$ is a singleton, say, $\operatorname{dom} \widetilde{T}=\left\{x_{0}\right\}$. In this case, the operator

$$
x \mapsto \begin{cases}(-\infty,+\infty) & \text { if } x=x_{0}, \\ \emptyset & \text { otherwise }\end{cases}
$$

defines a maximal monotone extension of $\widetilde{T}$, and hence also defines a maximal monotone extension of $T$, which is contained in $\mathcal{T}$.

(iii) Suppose $\operatorname{int}(\operatorname{dom} \widetilde{T}) \neq \emptyset$. Then $0 \in \operatorname{int}\left(\operatorname{dom} \widetilde{T}_{1}-\operatorname{dom} \widetilde{T}_{2}\right)$ and hence, by Fact 2.3 , the extension $\widetilde{T}$ is maximal monotone. Let $\left\{b_{i}\right\}_{i=1}^{l}$ denote the union of the sets of breakpoints for $\widetilde{T}_{1}$ and $\widetilde{T}_{2}$, provided by Definition 3.2. To see that $\widetilde{T} \in \mathcal{T}$, observe that the restriction of $\widetilde{T}$ to each open interval $\left(b_{i}, b_{i+1}\right)$ is either single-valued and continuous, single-valued and strictly monotone, or identically equal to the empty-set.

(c): Let $\widetilde{T} \in \mathcal{T}(B)$ be a maximal monotone extension of $T$. Since $\widetilde{T}^{-1}$ is a maximal monotone extension of $T^{-1}$, it suffices to show that $\widetilde{T}^{-1} \in \mathcal{T}$. To this end, observe that

$$
\operatorname{dom} \widetilde{T}^{-1}=\operatorname{range} \widetilde{T}=\left(\bigcup_{i=0}^{l} \widetilde{T}\left(\left(b_{i}, b_{i+1}\right)\right)\right) \cup\left(\bigcup_{i=1}^{l-1} \widetilde{T}\left(b_{i}\right)\right),
$$

where we denote $\widetilde{T}\left(\left(b_{i}, b_{i+1}\right)\right):=\left\{y \in \widetilde{T}(x): x \in\left(b_{i}, b_{i+1}\right)\right\}$. Both $\widetilde{T}$ and $\widetilde{T}^{-1}$ are maximal monotone and closed convex-valued [22, Exerc.12.8]. To show that $\widetilde{T}^{-1} \in$ $\mathcal{T}$, it suffices to show that on each piece of its domain specified by (6) which is not a singleton, that $\widetilde{T}^{-1}$ is single-valued and either constant, or strictly monotone and hence continuous by maximal monotonicity. To see this, we distinguish the following cases, using the fact that $\widetilde{T} \in \mathcal{T}$. 
(i) Consider a piece in (6) of the form $\widetilde{T}\left(\left(b_{i}, b_{i+1}\right)\right)$. There are thus two possibilities:

(I) $\widetilde{T}$ is single-valued and constant with value $c$ on $\left(b_{i}, b_{i+1}\right)$ : In this case $\widetilde{T}^{-1}(c)$ is a closed interval containing $\left(b_{i}, b_{i+1}\right)$.

(II) $\widetilde{T}$ is single-valued, continuous and strictly monotone on $\left(b_{i}, b_{i+1}\right)$ : In this case, $\widetilde{T}^{-1}$ is single-valued, continuous and strictly monotone on $U=$ $\widetilde{T}\left(\left(b_{i}, b_{i+1}\right)\right)$ and, moreover, $U$ is an interval [23, Th. 5.11.14].

(ii) Next consider a piece in (6) of the form $\widetilde{T}\left(b_{i}\right)$ where $b_{i} \in \operatorname{dom} \widetilde{T}$. Then $b_{i} \in$ $\widetilde{T}^{-1}(y)$ for all $y \in \widetilde{T}\left(b_{i}\right)$ where $\widetilde{T}\left(b_{i}\right)$ is a closed convex set due to the maximal monotonicity of $\widetilde{T}$. If $\operatorname{int} \widetilde{T}\left(b_{i}\right)=\emptyset$ then $\widetilde{T}\left(b_{i}\right)$ is a singleton and there is nothing further to prove. Suppose, then, that the open interval $U:=\operatorname{int} \widetilde{T}\left(b_{i}\right)$ is non-empty. There are two possibilities.

(I) $\widetilde{T}^{-1}$ is single-valued on $U$ : Then $\widetilde{T}^{-1}(y)=\left\{b_{i}\right\}$ for all $y \in U$.

(II) $\widetilde{T}^{-1}$ is multi-valued on $U: \mathrm{m}$ Then there exist points $y_{0} \in U$ and $x_{0} \neq b_{i}$ such that $x_{0} \in \widetilde{T}^{-1}\left(y_{0}\right)$ and $y_{0}>y \geq \inf \widetilde{T}\left(b_{i}\right)$. For convenience, we assume that $x_{0}<b_{i}$; an analogous argument applies when $x_{0}>b_{i}$. Since $\widetilde{T}^{-1}$ is closed and convex-valued, $\left[x_{0}, b_{i}\right] \subseteq \widetilde{T}^{-1}\left(y_{0}\right)$ and hence $y_{0} \in \widetilde{T}(x)$ for all $\underset{\widetilde{T}}{x} \in\left[x_{0}, b_{i}\right]$. Since $\widetilde{T} \in \mathcal{T}$, it must be single-valued on $\left(b_{i-1}, b_{i}\right)$, hence $\widetilde{T} x=\left\{y_{0}\right\}$ for all $x \in\left(b_{i-1}, b_{i}\right)$. Since $\widetilde{T}$ is maximal monotone, it must hold that $y_{0}=\inf T\left(b_{i}\right) \leq \underline{y}$ which is a contradiction.

We conclude, therefore, that $\widetilde{T}^{-1}(y)=\left\{b_{i}\right\}$ for all $y \in \widetilde{T}\left(b_{i}\right)$.

Cases (i) and (ii) together imply that $\widetilde{T}^{-1} \in \mathcal{T}$ which completes the proof of (c).

(d): By (a) it follows that $\lambda T \in \mathcal{T}$. Noting that the identity operator $I$ is a maximal monotone operator contained in $\mathcal{T}$ with $\operatorname{dom} I=\mathbb{R}$, (b) implies that $I+\lambda T \in \mathcal{T}$. The result now follows from (c).

(e): This follows immediately from parts (b) and (c).

Example 3.1 (Examples of $\mathcal{T}$-operators) Proposition 3.3(d) ensures that the resolvent of any $\mathcal{T}$-operator belongs to $\mathcal{T}$. In particular, $\mathcal{T}$ contains all proximity mappings of $\mathcal{F}$-functions. In other words, if $f \in \mathcal{F}$ and $\lambda>0$ then

$$
\operatorname{prox}_{f}^{\lambda}:=\underset{y \in \mathbb{R}}{\arg \min }\left\{f(y)+\frac{1}{2 \lambda}\|\cdot-y\|^{2}\right\}=(I+\lambda \partial f)^{-1} \in \mathcal{T} .
$$

In particular, by considering the indicator function contained in $\mathcal{F}$, we see that $\mathcal{T}$ contains all projection operators onto closed, convex subsets of $\mathbb{R}$.

Remark 3.3 (Maximal monotone extensions of $T \in \mathcal{T}$ ) When defining the class of $\mathcal{T}$-operators in Definition 3.2, one might have instead required an operator $T \in \mathcal{T}$ to be maximal monotone itself rather than its extension in $\mathcal{T}$. This approach has a significant shortcoming in that the empty relation is no longer in $\mathcal{T}$. Consequently, Proposition 3.3(b) no longer holds as can be seen by considering the sum two maximal monotone operators whose domains do not intersect.

The following theorem summarizes the connection between $\mathcal{F}$-functions and $\mathcal{T}$-operators.

Theorem 3.1 If $f \in \mathcal{F}$ is proper then $\partial f$ is maximal monotone and belongs to $\mathcal{T}$. Conversely, if $T \in \mathcal{T}$ is maximal monotone then there exists a proper, lsc, convex function $f$ such that $T=\partial f$ and, moreover, any such function belongs to $\mathcal{F}$. 
Proof If $f \in \mathcal{F}$ is a proper function then the fact that $\partial f$ is maximal monotone and belongs to $\mathcal{T}$ was already proven in Proposition 3.2.

Conversely, let $T \in \mathcal{T}$ be a maximal monotone operator. By Fact 2.2, there exists at least one proper, lsc, convex function with subdifferential equal to $T$. Let $f$ denote any such function (which already satisfies Definition 3.1(a)). By Fact 2.4, $f$ is continuous on $\operatorname{dom} f$, that is, Definition 3.1(b) is satisfied. Finally, to show that $f$ satisfies Definition 3.1(c), first recall that a convex function is differentiable at point in its domain if and only if its subdifferential is a singleton at the same point [11, Th. 2.2.1]. It follows that $f$ can be non-differentiable only if $T$ is multivalued which happens at most at finitely many points. Consider the restriction of the function $f$ to an open interval on which it is differentiable. Then, as $T \in \mathcal{T}$, we have that $f^{\prime}$ is either constant or strictly monotone on this interval. If $f^{\prime}$ constant then $f$ is affine. Otherwise $f^{\prime}$ is strictly monotone and hence $f$ is strictly convex [22, Th. 2.13].

Note that Theorem 3.1 provides a pathway to symbolically computing a maximal monotone extension of a monotone operators $T \in \mathcal{T}$. First find function $f \in \mathcal{F}$ such that $\partial f=T$. A maximal extension of $T$ is then given by $\partial f$.

We now return to the question closure of $\mathcal{F}$-function under the operation of Fenchel conjugation. We offer the following proof, which utilizes our class of monotone operators.

Proposition 3.4 ( $\mathcal{F}$ is closed under Fenchel conjugation) If $f \in \mathcal{F}$ is proper then $f^{*} \in \mathcal{F}$.

Proof By Theorem 3.1, $\partial f \in \mathcal{T}$. Combining Fact 2.1 with Proposition 3.3(c) shows that $\partial f^{*}=(\partial f)^{-1} \in \mathcal{T}$. Using Theorem 3.1 a second time yields $f^{*} \in \mathcal{F}$.

Assumption (c) of Definition 3.1 is crucial for obtaining closedness of the family $\mathcal{F}$ under Fenchel conjugation. Specifically, the strict convexity assumption on non-constant pieces of the domain cannot be removed and replaced with mere differentiability. In fact, the following counter-example shows that this is the case even for infinitely differentiable convex functions.

Example 3.2 (Necessity of finitely affine pieces) Consider the convex function (see Figure 1) constructed from (unnormalized) $C^{\infty}$ mollifying functions as defined follows:

$$
f(x):=\int_{0}^{x} \int_{0}^{y} h(z) d z d y
$$

with

$$
h(x):= \begin{cases}\psi\left(2^{2 n+1} x-1\right) & \text { for } x \in\left[2^{-(2 n+1)}, 2^{-2 n}\right] \quad\left(n \in \mathbb{N}_{0}\right) \\ 0 & \text { for } x \in\left(2^{-(2 n+2)}, 2^{-(2 n+1)}\right) \quad\left(n \in \mathbb{N}_{0}\right) \\ 0 & \text { for } x \in(-\infty, 0] \cup(1, \infty),\end{cases}
$$

where $\psi$ denotes the mollifying function given by

$$
\psi(x):= \begin{cases}\exp \left(-\frac{1}{1-(2 x-1)^{2}}\right) & x \in[0,1] \\ 0 & \text { else. }\end{cases}
$$




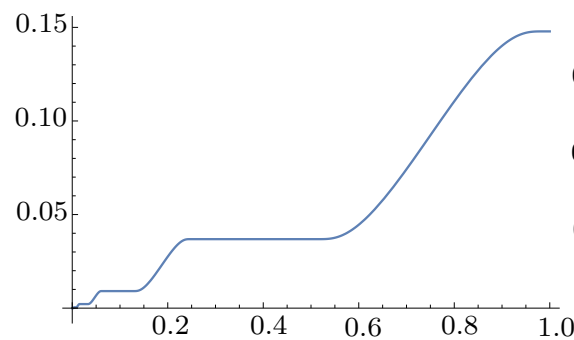

(a) The monotone operator $f^{\prime}$ on $[0,1]$.

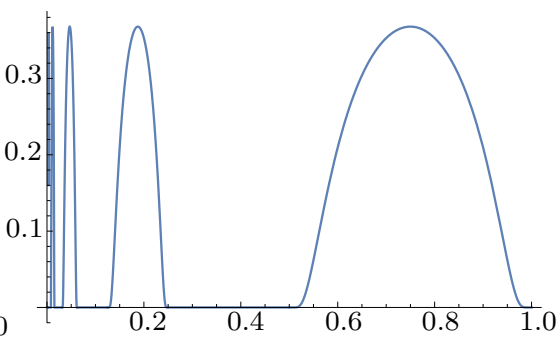

(b) The function $h=f^{\prime \prime}$ on $[0,1]$.

Fig. 1: Construction of the convex function $f$ in $(7)$.

The function $h$ is nonnegative and infinitely differentiable on $\mathbb{R} \backslash\{0\}$ so that $\int_{0}^{y} h(z) d z$ is continuous nondecreasing. It follows that $f$ is convex on $\mathbb{R}$ and infinitely differentiable on $\mathbb{R} \backslash\{0\}$ and hence satisfies properties (a)-(b) of Definition 3.1. The function $f$, however does not satisfy (c) of Definition 3.1 as it is affine on every interval $\left(2^{-(2 n+2)}, 2^{-(2 n+1)}\right)$ for $n \in \mathbb{N}_{0}$ fixed with slope $a_{n}$ given by

$$
\begin{aligned}
a_{n}=\int_{0}^{2^{-2(n+1)}} h(z) d z & =\sum_{j=n+1}^{\infty} \int_{\mathbb{R}} \psi\left(2^{2 j+1} x-1\right) d x \\
& =\sum_{j=n+1}^{\infty} 2^{-(2 j+1)} \int_{\mathbb{R}} \psi(y) d y .
\end{aligned}
$$

Now, since $f$ is affine on infinitely many intervals in $[0,1 / 2]$, its subdifferential, $\partial f$, is constant and singleton on infinitely many intervals in $[0,1 / 2]$ with value on these intervals given by $\left\{a_{n}\right\}_{\mathbb{N}_{0}}$. It follows that $\partial f^{*}=(\partial f)^{-1}$ is multi-valued at each point in $\left\{a_{n}\right\}_{\mathbb{N}_{0}}$, of which there are infinitely many, and therefore $f^{*}$ cannot be in $\mathcal{F}$.

\section{Examples and Illustrations}

In this section we detail a number of computational examples and applications which utilize the class of monotone operators introduced above. We perform our symbolic computations in Maple making use of the data-structures provided by the Symbolic Convex Analysis Toolkit (SCAT) developed by Borwein \& Hamilton [10]. We shall also make use of an additional function, shown in Figure 2, for computing the inverse of a monotone operator. The source code for the examples which follow as well as the SCAT library are available online at:

$$
\text { http://vaopt.math.uni-goettingen.de/software.php }
$$

Although we consider the one-dimensional setting, it is worth noting that, as recognized by [7], separable convex functions on $\mathbb{R}^{n}$ can still be handled. Recall that a convex function $f: \mathbb{R}^{n} \rightarrow(-\infty,+\infty]$ is separable if there exist convex functions $f_{j}: \mathbb{R} \rightarrow(-\infty,+\infty]$ such that $f(x)=\sum_{j=1}^{n} f_{j}\left(x_{j}\right)$. For such a function,

$$
\partial f(x)=\partial f_{1}\left(x_{1}\right) \times \cdots \times \partial f_{n}\left(x_{n}\right), \quad f^{*}(y)=\sum_{j=1}^{n} f_{j}^{*}\left(y_{j}\right) .
$$


In this way, we may also consider monotone operators $T: \mathbb{R}^{n} \rightrightarrows \mathbb{R}^{n}$ such that

$$
T(x)=T_{1}\left(x_{1}\right) \times \cdots \times T_{n}\left(x_{n}\right)
$$

where each $T_{j}: \mathbb{R} \rightrightarrows \mathbb{R}$ is a monotone operator. We give examples in which this kind of structure arising in Sections 4.1 and 4.2.

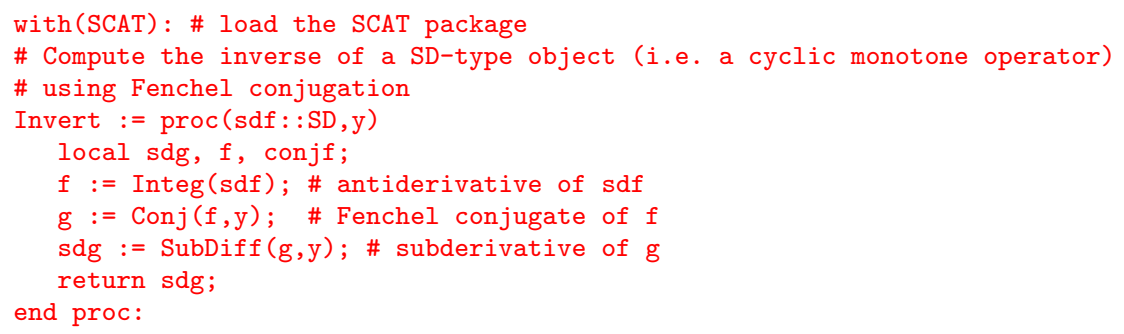

Fig. 2: Inversion of a maximal cyclic monotone operator using Fenchel conjugation.

\subsection{Explicit Formula for Proximity Operators}

Recall that the proximity operator of a proper, lsc, convex function $f: \mathbb{R}^{n} \rightarrow$ $(-\infty,+\infty]$ with parameter $\lambda>0$ is given by

$$
\operatorname{prox}_{f}^{\lambda}:=\underset{y \in \mathbb{R}}{\arg \min }\left\{f(y)+\frac{1}{2 \lambda}\|\cdot-y\|^{2}\right\}
$$

Proximity operators are the building blocks of many iterative algorithms in optimization and thus it is important, in practice, that they can be efficiently computed. One such possibility is to find an explicit formula for the proximity operator.

We compute explicit forms for some classical proximity operators using the framework of $\mathcal{T}$-operators. Our approach exploits the formula

$$
\operatorname{prox}_{f}^{\lambda}=(I+\lambda \partial f)^{-1}
$$

Given a function $f \in \mathcal{F}$, we symbolically compute its subdifferential using the SCAT package. Using (8) and Proposition 3.3, we deduce that $\operatorname{prox}_{f}^{\lambda} \in \mathcal{T}$.

Two example computations of proximity operators are in given in Figures 3 and 4 . Furthermore, it is worth nothing that, thanks to our theory in Section 3, this is computation actually proves the resulting formula for the proximity function. 
with(SCAT): \# load the SCAT package

$f:=\operatorname{convert}(\operatorname{abs}(x), P W F)$; \# define $f$ and convert to PWF format

$$
f:= \begin{cases}-x & x<0 \\ 0 & x=0 \\ x & x>0\end{cases}
$$

sdf := SubDiff(f): \# compute the subdiff of $f$ Assume (lambda >0):

prox ['f'] ${ }^{\wedge}$ lambda $=\operatorname{Invert}(\operatorname{simplify}(x+l a m b d a * s d f), y) ; \#$ prox as fn of $y$

$$
\operatorname{prox}_{f}^{\lambda}= \begin{cases}\{\lambda+y\} & y<-\lambda \\ \{0\} & y=-\lambda \\ \{0\} & (-\lambda<y) \text { and }(y<\lambda) \\ \{0\} & y=\lambda \\ \{-\lambda+y\} & \lambda<y\end{cases}
$$

Fig. 3: Computation of the proximity function of $f=\|\cdot\|_{1}$.

with(SCAT): \# load the SCAT package

Assume $(a<b)$ : \# assume that the interval is proper

$\mathrm{f}:=\operatorname{convert}($ piecewise $(\mathrm{a}<=\mathrm{x}$ and $\mathrm{x}<=\mathrm{b}, 0$, infinity), PWF, $\mathrm{x})$;

$$
f:= \begin{cases}\infty & x<a \\ 0 & x=a \\ 0 & (a<x) \text { and }(x<b) \\ 0 & x=b \\ \infty & b<x\end{cases}
$$

sdf := SubDiff(f): \# compute the subdiff of $f$

$\mathrm{P}[\mathrm{a}, \mathrm{b}]]=\operatorname{Invert}(\operatorname{simplify}(\mathrm{x}+\mathrm{sdf}), \mathrm{y})$; \# projector onto $[\mathrm{a}, \mathrm{b}]$ as fn of $\mathrm{y}$

$$
P_{[a, b]}= \begin{cases}\{a\} & x<a \\ \{a\} & x=a \\ \{y\} & (a<y) \text { and }(y<b) \\ \{b\} & x=b \\ \{b\} & b<x\end{cases}
$$

Fig. 4: Computation of the proximity function of $f=\iota_{[a, b]}$ (i.e., projector onto $[a, b])$.

\subsection{Recovery of Penalty Functions}

Given a monotone operator $T: \mathbb{R}^{n} \rightrightarrows \mathbb{R}^{n}$, we consider the problem of finding a so-called penalty function $f: \mathbb{R}^{n} \rightarrow(-\infty,+\infty]$, that is, a function $f$ whose subdifferential can be identified with $T$. Precisely, find a function $f$ such that

$$
\operatorname{gph} T \subseteq \operatorname{gph}\left(\operatorname{prox}_{f}^{\lambda}\right)
$$


We shall focus on the case in which $\lambda=1$ as it covers all the technicalities of the general case and thus we define $\operatorname{prox}_{f}:=\operatorname{prox}_{f}^{1}$. The same problem was previously studied in [9], without symbolic computational tools.

The following proposition and its proof shall form the basis of our approach.

Proposition 4.1 (Recovery of penalty functions) Let $T: \mathbb{R}^{n} \rightrightarrows \mathbb{R}^{n}$ be a maximal cyclically monotone operator. There exists a proper, lsc function $f$ with $f+\frac{1}{2}\|\cdot\|^{2}$ convex such that $T=\operatorname{prox}_{f}$. Furthermore, if $T \in \mathcal{F}$ then there exists an $f$ such that $f+\frac{1}{2}\|\cdot\|^{2}$ belongs to $\mathcal{F}$.

Proof By Fact 2.2, there exists a proper, lsc, convex function $h: \mathbb{R}^{n} \rightarrow(-\infty,+\infty]$ such that $T=\partial h$, and in particular, if $T \in \mathcal{T}$ then Theorem 3.1 ensures that we may choose $h \in \mathcal{F}$. Using Fermat's rule [22, Th. 10.1], we deduce that

$$
\begin{aligned}
T(x)=\partial h(x)=\left(\partial h^{*}\right)^{-1}(x) & =\left\{y \in \mathbb{R}^{n}: x \in \partial h^{*}(y)\right\} \\
& =\underset{y \in \mathbb{R}^{n}}{\arg \min }\left\{h^{*}(y)-\langle x, y\rangle\right\} \\
& =\underset{y \in \mathbb{R}^{n}}{\arg \min }\left\{\left(h^{*}(y)-\frac{1}{2}\|y\|^{2}\right)+\frac{1}{2}\|x-y\|^{2}\right\} .
\end{aligned}
$$

We therefore have $T=\operatorname{prox}_{f}$ where $f:=h^{*}-\frac{1}{2}\|\cdot\|^{2}$. The fact that $f$ is proper and lsc with $f+\frac{1}{2}\|\cdot\|^{2}$ convex follows since $h^{*}$ is proper, lsc and convex. In particular, if $h \in \mathcal{F}$ then $f+\frac{1}{2}\|\cdot\|^{2}=h^{*} \in \mathcal{F}$.

We are now ready to state our strategy for reconstruction of the penalty function associated with the monotone operator $T \in \mathcal{T}$.

(i) Find a maximal cyclically monotone extension of $\widetilde{T} \in \mathcal{T}$ of $T$.

(ii) Find a function $h \in \mathcal{F}$ such that $\partial h=\widetilde{T}$.

(iii) Compute the Fenchel conjugate $h^{*}$ of $h$.

(iv) The penalty function $f$ can now be given as $f:=h^{*}-\frac{1}{2}\|\cdot\|^{2}$.

We note that the existence of a function $h$ in Step (ii) is possible due to Theorem 3.1 and can be obtained via integrating any selection of $T$ [17, Prop. 1.6.1]. Step (iii) can be performed for $\mathcal{F}$-functions within the SCAT package and Step (iv) is clearly straightforward. Thus the only potentially difficult computation arises in Step (i); but this can sometimes be dealt with satisfactorily as we shall now demonstrate.

Example 4.1 (Hidden convexity of the hard thresholding operator) The hard thresholding operator $H_{\alpha}: \mathbb{R} \rightarrow \mathbb{R}$ for parameter $\alpha>0$ is defined by

$$
H_{\alpha}(x):= \begin{cases}x & \text { if }|x|>\alpha \\ 0 & \text { otherwise }\end{cases}
$$

In compressive sensing, $H_{\alpha}$ is usually viewed as a selection of the (set-valued) proximity operator of the $\ell_{0}$-functional; a non-convex object. More precisely,

$$
\operatorname{prox}_{\frac{\alpha}{2}\|\cdot\|_{0}}(x)=\underset{y \in \mathbb{R}}{\arg \min }\left\{\alpha\|y\|_{0}+|x-y|^{2}\right\}= \begin{cases}x & \text { if }|x|>\alpha, \\ \{0, x\} & \text { if }|x|=\alpha, \\ 0 & \text { otherwise; }\end{cases}
$$


and hence that gph $H_{\alpha} \subseteq$ gph prox $\frac{\alpha}{2}\|\cdot\|_{0}$. Whilst both $H_{\alpha}$ and $\operatorname{prox}_{\frac{\alpha}{2}\|\cdot\|_{0}}$ are monotone operators, neither are maximal. Nevertheless, on account of having full domain, their unique maximal monotone extension can be given

$$
T(x):= \begin{cases}x & \text { if }|x|>\alpha \\ {[0, x]} & \text { if }|x|=\alpha, \\ 0 & \text { otherwise }\end{cases}
$$

and, moreover, it is easy to verify that $T$ belongs to $\mathcal{T}$.

We are now in a position to recover the penalty function $f$ associated with $T$.

with(SCAT): \# load the SCAT package

Assume (alpha >0):

$\mathrm{H}:=\mathrm{SD}([\mathrm{x}$, -alpha, $0, \mathrm{x}, 0$, alpha, $0, \mathrm{x}, \mathrm{x}],[\mathrm{x}], \mathrm{x}:$ :real $)$;

$$
H:= \begin{cases}\{x\} & x<-\alpha \\ \{0, x\} & x=-\alpha \\ \{0\} & (-\alpha<x) \text { and }(x<\alpha) \\ \{0, x\} & x=\alpha \\ \{x\} & x<\alpha\end{cases}
$$

$\operatorname{Conjh}:=\operatorname{Conj}(\operatorname{Integ}(\mathrm{H}), \mathrm{y})$

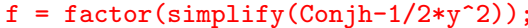

$$
f= \begin{cases}0 & y<-\alpha \\ \frac{1}{2}(\alpha-y)(\alpha+y) & y=-\alpha \\ \frac{1}{2}(\alpha+y)^{2} & (-\alpha<y) \text { and }(y<0) \\ -\frac{1}{2} \alpha^{2}-\frac{1}{2} y^{2} & y=0 \\ -\frac{1}{2}(\alpha-y)^{2} & (0<y) \text { and }(y<\alpha) \\ \frac{1}{2}(\alpha-y)(\alpha+y) & y=\alpha \\ 0 & \alpha<y\end{cases}
$$

Fig. 5: Recovery of a convex penalty associated with $H_{\alpha}$.

A closer look at the result from Figure 5 shows that the penalty function $f$ may be expressed more concisely in the form

$$
f(y)= \begin{cases}0 & |y|>\alpha, \\ -\frac{1}{2}(|y|-\alpha)^{2} & |y| \leq \alpha .\end{cases}
$$

It is also worth noting, that the entire procedure can be reserved to give a proof that the hard thresholding operator is monotone! More precisely, one should symbolically compute the proximity function of $f$ in (9) using the method in Section 4.1. If the result is an extension of the original operator, $H_{\alpha}$, then it is necessarily monotone (as the subdifferential of a proper, lsc, convex function). 
4.3 Superexpectations, superdistributions and superquantiles

The class of functions $\mathcal{T}$ is well-suited for direct symbolic calculation of superexpectations, superdistributions and superquantiles as developed in $[18,13,19]$. The superexpectation function, $\overline{\mathbf{E}}_{X}(x)$, of the random variable $X$ at level $x$ is defined as

$$
\begin{aligned}
\mathbf{E}_{X}(x):=\mathbb{E}[\max \{x, X\}] & =\int_{-\infty}^{\infty} \max \left\{x, x^{\prime}\right\} d F_{X}\left(x^{\prime}\right) \\
& =\int_{0}^{1} \max \left\{x, Q_{X}(p)\right\} d p
\end{aligned}
$$

where $F_{X}: \mathbb{R} \rightarrow[0,1]$ is the cumulative distribution function of the random variable $X$ and $Q_{X}:(0,1) \rightarrow(-\infty,+\infty)$ is the quantile function; these are defined respectively as

$$
\begin{aligned}
& F_{X}(x):=\operatorname{prob}\{X \leq x\} \\
& Q_{X}(p):=\min \left\{x \mid F_{X}(x) \geq p\right\} \quad(p \in(0,1)) .
\end{aligned}
$$

The function $F_{X}$ is nondecreasing and right-continuous on $(-\infty,+\infty)$ with

$$
\lim _{x \rightarrow-\infty} F_{X}(x)=0, \quad \lim _{x \rightarrow+\infty} F_{X}(x)=1 .
$$

The maximal monotone extension of the distribution function $F_{X}$, denoted $\widetilde{\mathcal{F}}$ is called the superdistribution and is also generated by taking the subdifferential of the superexpectation function [19, Th. 1], which is a finite convex function on $(-\infty, \infty)$ with

$$
\operatorname{gph} \widetilde{\mathcal{F}}_{X}=\operatorname{gph} \partial \mathbf{E}_{X}, \quad F_{X}(x)=\mathbf{E}_{X}^{\prime+}(x),
$$

where

$$
\mathbf{E}_{X}^{\prime+}(x):=\lim _{y \downarrow x} \frac{\mathbf{E}_{X}(y)-\mathbf{E}_{X}(x)}{y-x} .
$$

The superquantile function, denoted $\widetilde{\mathcal{Q}}_{X}$ is the maximal monotone extension of the quantile function and satisfies the inverse relationship [19, Th. 2]

$$
\left(\operatorname{gph} \widetilde{\mathcal{F}}_{X}\right)^{-1}=\operatorname{gph} \partial \mathbf{E}_{X}^{*}=\operatorname{gph} \widetilde{\mathcal{Q}}_{X}, \text { and } Q_{X}(p)=\mathbf{E}_{X}^{*^{\prime}-}(p) .
$$

These objects are therefore amenable to symbolic convex analysis, via subdifferentials of the superexpectation function $\mathbf{E}_{X}$ or its Fenchel conjugate. This provides a symbolic route to working with coherent risk measures such as conditional valuedat-risk.

To demonstrate this approach, we symbolically derive an example which appears in [19].

Example 4.2 (Exponential distributions) Let $X$ be exponentially distributed with parameter $\lambda>0$. That is, $X$ has cumulative distribution function $F_{X}=1-\exp (-\lambda x)$. Figure 6 shows the symbolic computation of the superexpectations function, the superdistribution function, and the superquantile function of $X$. Note that, to compute the superexpection of $F_{X}$, we have made use of the fact that

$$
\lim _{x \rightarrow \infty}\left[E_{X}(x)-x\right]=0,
$$

which was proven as part of $[19$, Th. 1]. In this way, it is not necessary to compute the potentially tricky ' $m a x$ ' in the definition (10) directly. 
with (SCAT) :

$\mathrm{F}:=1-\exp (-\operatorname{lambda} * \mathrm{x}):$ \# distribution $\mathrm{fn}$ of $\mathrm{X}$

$Q:=\operatorname{solve}(F=p, x)$; \# quantile $f n$ of $X$

$$
Q:=-\frac{\ln (1-p)}{\lambda}
$$

$\operatorname{superQ}:=\operatorname{factor}(1 /(1-p) * \operatorname{int}(\operatorname{subs}(p=t, Q), t=p . .1)) ; \#$ superquantile fn of $X$

$$
\operatorname{super} Q:=-\frac{\ln (1-p)-1}{\lambda}
$$

Assume (lambda>0):

$\mathrm{F}:=\operatorname{convert}($ piecewise $(\mathrm{x}>=0, \mathrm{~F}), \mathrm{SD}, \mathrm{x})$;

$$
E:= \begin{cases}\{0\} & x<0 \\ \{0\} & x=0 \\ \left\{1-e^{-\lambda x}\right\} & 0<x\end{cases}
$$

\# compute the superexpection function of $\mathrm{F}$

E0 := Integ $(\mathrm{F}, \mathrm{x})$ :

c0 := Eval (simplify $(E 0-x), x$ = infinity):

$\mathrm{E}:=\operatorname{simplify}(\mathrm{EO}-\mathrm{cO})$;

$$
E:= \begin{cases}\frac{1}{\lambda} & x<0 \\ \frac{1}{\lambda} & x=0 \\ \frac{\lambda x+e^{-\lambda x}}{\lambda} & 0<x\end{cases}
$$

$\operatorname{conjE}:=\operatorname{conjE}(E, p, x) ; \#$ the conjugate of the superexpectation

$$
\operatorname{conj} E:= \begin{cases}\infty & p<0 \\ -\frac{1}{\lambda} & p=0 \\ -\frac{(-1+p)(\ln (1-p)-1)}{\lambda} & (0<p) \text { and }(p<1) \\ 0 & p=1 \\ \infty & 1<p\end{cases}
$$

Fig. 6: Computation of super-functions for the exponential distribution.

Acknowledgements DRL was supported in part by Deutsche Forschungsgemeinschaft Collaborative Research Center SFB755. MKT was supported by Deutsche Forschungsgemeinschaft RTG2088.

\section{References}

1. B. Gardiner, J.K., Lucet, Y.: Conjugate of convex piecewise linear-quadratic bivariate functions. Comput. Optim. Appl. 58, 249-272 (2014). DOI 10.1007/s10589-013-9622-z

2. Bailey, D.H., Borwein, J.M.: Mathematics by Experiment: Plausible Reasoning in the 21st century. A K Peters Ltd, Natick, MA (2003)

3. Bailey, D.H., Borwein, J.M.: Experimental Mathematics: examples, methods and implications. Notices Amer. Math. Soc. 52(5), 502-514 (2005) 
4. Bailey, D.H., Borwein, J.M., Calkin, N.J., Girgensohn, R., Luke, D.R., Moll, V.H.: Experimental Mathematics in Action. A K Peters Ltd, Natick, MA (2007)

5. Bailey, D.H., Borwein, J.M., Girgensohn, R.: Experimentation in Mathematics: Computational Paths to Discovery. A K Peters Ltd, Natick, MA (2003)

6. Bauschke, H.H., Combettes, P.L.: Convex Analysis and Monotone Operator Theory in Hilbert Spaces. Springer Science \& Business Media (2011)

7. Bauschke, H.H., von Mohrenschildt, M.: Fenchel conjugates and subdifferentials in Maple. Tech. rep. (1997)

8. Bauschke, H.H., von Mohrenschildt, M.: Symbolic computation of Fenchel conjugates. ACM Commun. Comput. Algebra 40(1), 18-28 (2006)

9. Bayram, I.: Penalty functions derived from monotone mappings. IEEE Signal Process. Lett. 22(3), 264-268 (2015)

10. Borwein, J.M., Hamilton, C.H.: Symbolic Fenchel conjugation. Math. Program. 116(1-2), 17-35 (2009)

11. Borwein, J.M., Vanderwerff, J.D.: Convex Functions: Constructions, Characterizations and Counterexamples, vol. 32. Cambridge University Press Cambridge (2010)

12. Burachik, R., Iusem, A.N.: Set-valued mappings and enlargements of monotone operators, vol. 8. Springer Science \& Business Media (2007)

13. Dentcheva, D., Martinez, G.: Two-stage stochastic optimization problems with stochastic ordering constraints on the recourse. Eur. J. Oper. Res. 219, 1-8 (2012)

14. Hamilton, C.H.: Symbolic convex analysis. Master's thesis, Simon Fraser University (2005)

15. Lucet, Y.: Faster than the fast Legendre transform, the linear-time Legendre transform. Numer. Algorithms 16(2), 171-185 (1997)

16. Lucet, Y.: What shape is your conjugate? a survey of computational convex analysis and its applications. SIAM Rev. 52(3), 505-542 (2010)

17. Niculescu, C., Persson, L.E.: Convex functions and their applications: a contemporary approach. Springer Science \& Business Media (2006)

18. Ogryczak, W., Ruszczyński, A.: Dual stochastic dominance and related mean-risk models. SIAM J. Optim. 13, 60-78 (2002)

19. Rockafellar, R., Royset, J.: Random variables, monotone relations, and convex analysis. Math. Program. (2014). DOI 10.1007/s10107-014-0801-1

20. Rockafellar, R.T.: Convex Analysis. Princeton University Press, Princeton, NJ (1970)

21. Rockafellar, R.T.: On the maximal monotonicity of subdifferential mappings. Pacific J. Math. 33, 209-216 (1970)

22. Rockafellar, R.T., Wets, R.J.: Variational Analysis. Grundlehren Math. Wiss. SpringerVerlag, Berlin (1998)

23. Vakil, N.: Real Analysis Through Modern Infinitesimals. Encyclopedia of Mathematics and its Applications, Cambridge University Press, New York (2011). 